\title{
A BREAF SURVEY ON ARMENDARIZ AND CENTRAL ARMENDARIZ RINGS
}

\author{
DUŠAN JOKANOVIĆ* \\ University of East Sarajevo, Production and Management Faculty Trebinje \\ *Corresponding author. E-mail: dusan.jokanovic@fpm.ues.rs.ba
}

DOI: 10.20948/mathmontis-2019-46-1

Summary. In this paper $R$ is a ring with unity, and $\sigma$ is endomorphism of the ring. We deal with central Armendariz rings as a natural generalization of Armendariz rings. We investigate a posibility of extending central Armendariz property from a ring to coresponding polynomial or matrix extension. At the end of this paper we consider an interesting note on reduced rings.

\section{INTRODUCTION}

Throughout this article $R$ denotes a ring with unity, $R[x]$ is coresponding polynomial ring, $\sigma$ denotes an endomorphism of $R, R[x ; \sigma]$ denotes skew polynomial ring with the ordinary addition and the multiplication subject to the relation $x r=\sigma(r) x$, and $R[[x ; \sigma]]$ denotes power series ring. The notion of Armendariz ring is introduced by Rege and Chhawchharia (see [2]). They defined a ring $R$ to be Armendariz if $f(x) g(x)=0$ implies $a_{i} b_{j}=0$, for all polynomials $f(x)=\sum_{i=0}^{n} a_{i} x^{i}$ and $g(x)=\sum_{j=0}^{m} b_{j} x^{j}$ from $R[x]$. The motivation for those rings comes from the fact that Armendariz had shown that the above reesult can be extended to a class of reduced rings, i.e., rings without non-zero nilpotent elements. In [1] authors introduced a class of central Armendariz rings. A ring $R$ is called central Armendariz ring if $f(x) g(x)=0$ implies $a_{i} b_{j} \in C(R)$, for all polynomials $f(x)=\sum_{i=0}^{n} a_{i} x^{i}$ and $g(x)=$ $\sum_{j=0}^{m} b_{j} x^{j}$ from $R[x]$, where $C(R)$ is center of a ring $R$. Clearly Armendariz rings are central Armendariz rings. It is known from [1] that a class of central Armendariz rings is closed for polynomial extensions and localizations, and that the central hhArmendariz rings are strictly between Armendariz rings and abelian rings. As a generalization of $\sigma$-skew Armendariz rings, Onyang (see [4]) introduced a notion of weak $\sigma$-skew Armendariz ring (see [3],[4].[5]). A weak $\sigma$-skew Armendariz ring $R$ is a ring in which $f(x) g(x)=0$ implies $a_{i} \sigma^{i}\left(b_{j}\right)$ is the nilpotent element of $R$ for all $f(x)=\sum_{i=0}^{n} a_{i} x^{i}$ and $g(x)=\sum_{j=0}^{m} b_{j} x^{j}$ from $R[x ; \sigma]$. Chain and Tong (see [5]) have proved that if $R$ and $S$ are rings and $\sigma$ is and isomorphism of rings $R$ and $S$ and $R$ is $\alpha$-skew Armendariz ring, then $S$ is $\sigma \alpha \sigma^{-1}$-skew Armendariz ring. In this paper we give (see [3]) a variant of this theorem for weak skew-Armendariz rings. In our main result we give an example of central Armendariz matrix ring $T(n, R)$, for reduced ring $R$.

\section{EXTENDING OF ARMENDARIZ PROPERTY}

In this section we deal with posibility of extending the Armendariz property under ring isomorphism (see [3]). From universal algebra we know that every homomorphism $\sigma$ of rings $R$ and $S$ can be extended to the homomorphism of the corresponding rings of polynomials $R[x]$ and $S[x]$ by $\sum_{i=0}^{m} a_{i} x^{i} \mapsto \sum_{i=0}^{m} \sigma\left(a_{i}\right) x^{i}$, which we also denote by $\sigma$. Chain and Tong in 
[5] prove that if $\sigma$ is ring isomorphism of $\operatorname{rings} R$ and $S$ and $R$ is $\alpha$-skew Armendariz, then $S$ is $\sigma \alpha \sigma^{-1}$ skew Armendariz ring. We prove the weak skew Armendariz variant of this theorem.

Theorem 2.1 ([3]) Let $R$ and $S$ be rings with a ring isomorphism $\sigma: R \rightarrow S$. If $R$ is weak $\alpha-$ skew Armendariz then $S$ is weak $\sigma \alpha \sigma^{-1}$-skew Armendariz.

Proof. Let $f(x)=\sum_{i=0}^{m} a_{i} x^{i}$ and $g(x)=\sum_{j=0}^{n} b_{j} x^{j}$ be polynomials from the ring $S\left[x ; \sigma \alpha \sigma^{-1}\right]$. We have to prove that $f(x) g(x)=0$ implies $a_{i}\left(\sigma \alpha \sigma^{-1}\right)^{i} b_{j} \in \operatorname{nil}(S)$, for all $i$ and $j$.

As we noted, $\sigma$ extends to the isomorphism of corresponding polynomial rings, so that there exist polynomials $f_{1}(x)=\sum_{i=0}^{m} a_{i}^{\prime} x^{i}$ and $g_{1}(x)=\sum_{j=0}^{m} b_{j}^{\prime} x^{j}$ from $R[x]$ such that $f(x)=\sigma\left(f_{1}(x)\right)=\sum_{i=0}^{m} \sigma\left(a_{i}^{\prime}\right) x^{i,}$ and $g(x)=\sigma\left(g_{1}(x)\right)=\sum_{j=0}^{m} \sigma\left(b_{j}^{\prime}\right) x^{j}$.

First, we shall show that $f(x) g(x)=0$ implies $f_{1}(x) g_{1}(x)=0$. If $f(x) g(x)=0$, we have

$$
a_{0} b_{k}+a_{1}\left(\sigma \alpha \sigma^{-1}\right)\left(b_{k-1}\right)+\ldots+a_{k}\left(\sigma \alpha \sigma^{-1}\right)^{k}\left(b_{0}\right)=0,
$$

for any $0 \leq k \leq m$. From the definition of $f_{1}(x)$ and $g_{1}(x)$, we have

$$
\sigma\left(a_{0}^{\prime}\right) \sigma\left(b_{k}^{\prime}\right)+\sigma\left(a_{1}^{\prime}\right)\left(\sigma \alpha \sigma^{-1}\right) \sigma\left(b_{k-1}^{\prime}\right)+\ldots+\sigma\left(a_{k}^{\prime}\right)\left(\sigma \alpha \sigma^{-1}\right)^{k} \sigma\left(b_{0}^{\prime}\right)=0,
$$

and using $\left(\sigma \alpha \sigma^{-1}\right)^{t}=\sigma \alpha^{t} \sigma^{-1}$ we obtain

$$
a_{0}^{\prime} b_{k}^{\prime}+a_{1}^{\prime} \alpha\left(b_{k-1}^{\prime}\right)+\ldots+a_{k}^{\prime} \alpha^{k}\left(b_{0}^{\prime}\right)=0,
$$

which means that $f_{1}(x) g_{1}(x)=0$ in the ring $R[x ; \alpha]$.

It remains to prove that $f_{1}(x) g_{1}(x)=0$ implies $a_{i}\left(\sigma \alpha \sigma^{-1}\right)^{i}\left(b_{j}\right) \in \operatorname{nil}(S)$. From the fact that $R$ is weak $\alpha$-skew Armendariz we have $a_{i}^{\prime} \alpha^{i}\left(b_{j}^{\prime}\right) \in \operatorname{nil}(R)$, and since

$$
a_{i}^{\prime}=\sigma^{-1}\left(a_{i}\right), b_{j}^{\prime}=\sigma^{-1}\left(b_{j}\right), \text { we have } \sigma^{-1}\left(a_{i}\right) \alpha^{i} \sigma^{-1}\left(b_{j}\right) \in \operatorname{nil}(R) .
$$

This implies

$$
\sigma^{-1}\left(a_{i}\right) \sigma^{-1} \sigma \alpha^{i} \sigma^{-1}\left(b_{j}\right)=\sigma^{-1}\left(a_{i}\left(\sigma \alpha \sigma^{-1}\right)^{i}\left(b_{j}\right)\right) \in \operatorname{nil}(R)
$$

and finally we obtain

$$
a_{i}\left(\sigma \alpha \sigma^{-1}\right)^{i}\left(b_{j}\right) \in \operatorname{nil}(S), 0 \leq i \leq m, 0 \leq j \leq n .
$$

Hence $S$ is weak $\sigma \alpha \sigma^{-1}$-skew Armendariz.

\section{MATRIX CENTRAL ARMENDARIZ RING $T(R, n)$}

In this section we give an example of matrix central Armendariz ring. For a ring $R$ consider a following set of triangular matrices 


$$
T_{n}(R)=\left\{\left[\begin{array}{lllll}
a_{11} & a_{12} & a_{13} & \ldots & a_{1 n} \\
0 & a_{22} & a_{23} & \ldots & a_{2 n} \\
0 & 0 & a_{33} & \ldots & a_{3 n} \\
\vdots & \vdots & \vdots & \ddots & \vdots \\
0 & 0 & 0 & \ldots & a_{n n}
\end{array}\right] \mid a_{i j} \in R\right\}
$$

We also consider the following set of triangular matrices over ring $R$

$$
T(R, n)=\left\{\left[\begin{array}{lllll}
a_{0} & a_{1} & a_{2} & \ldots & a_{n-1} \\
0 & a_{0} & a_{1} & \ldots & a_{n-2} \\
0 & 0 & a_{0} & \ldots & a_{3 n} \\
\vdots & \vdots & \vdots & \ddots & \vdots \\
0 & 0 & 0 & \ldots & a_{0}
\end{array}\right] \mid a_{i} \in R\right\}
$$

which is subring of $T_{n}(R)$. It is well known that $T_{n}(R)$ and $T(R, n)$ are subrings of the triangular matrix rings with matrix addition and multiplication. Let $\alpha$ be endomorphism of ring $R$. It is well known that endomorphism $\alpha$ can be naturally extended to an endomorphism

$$
\bar{\alpha}: T_{n}(R) \rightarrow T_{n}(R),
$$

and

$$
\bar{\alpha}: T(R, n) \rightarrow T(R, n)
$$

with:

$$
\bar{\alpha}\left(\left[\begin{array}{lllll}
a_{11} & a_{12} & a_{13} & \ldots & a_{1 n} \\
0 & a_{22} & a_{23} & \ldots & a_{2 n} \\
0 & 0 & a_{33} & \ldots & a_{3 n} \\
\vdots & \vdots & \vdots & \ddots & \vdots \\
0 & 0 & 0 & \ldots & a_{n n}
\end{array}\right]\right)=\left[\begin{array}{lllll}
\alpha\left(a_{11}\right) & \alpha\left(a_{12}\right) & \alpha\left(a_{13}\right) & \ldots & \alpha\left(a_{1 n}\right) \\
0 & \alpha\left(a_{22}\right) & \alpha\left(a_{23}\right) & \ldots & \alpha\left(a_{2 n}\right) \\
0 & 0 & \alpha\left(a_{33}\right) & \ldots & \alpha\left(a_{3 n}\right) \\
\vdots & \vdots & \vdots & \ddots & \vdots \\
0 & 0 & 0 & \ldots & \alpha\left(a_{n n}\right)
\end{array}\right]
$$

and

$$
\bar{\alpha}\left(\left[\begin{array}{lllll}
a_{0} & a_{1} & a_{13} & \ldots & a_{n-1} \\
0 & a_{0} & a_{1} & \ldots & a_{n-2} \\
0 & 0 & a_{33} & \ldots & a_{3 n} \\
\vdots & \vdots & \vdots & \ddots & \vdots \\
0 & 0 & 0 & \ldots & a_{0}
\end{array}\right]\right)=\left[\begin{array}{lllll}
\alpha\left(a_{0}\right) & \alpha\left(a_{1}\right) & \alpha\left(a_{2}\right) & \ldots & \alpha\left(a_{n-1}\right) \\
0 & \alpha\left(a_{0}\right) & \alpha\left(a_{1}\right) & \ldots & \alpha\left(a_{n-2}\right) \\
0 & 0 & \alpha\left(a_{33}\right) & \ldots & \alpha\left(a_{3 n}\right) \\
\vdots & \vdots & \vdots & \ddots & \vdots \\
0 & 0 & 0 & \ldots & \alpha\left(a_{0}\right)
\end{array}\right]
$$

Theorem 3.1 If $R$ is reduced ring then $T(R, n)$ is central Armendariz ring.

Proof. From [1] we obtain that for reduced ring $R$, the factor ring $R[x] /\left(x^{n}\right)$ is central Armendariz, for all $n \geq 2$. We use the ring isomorphism $f: R[x] /\left(x^{n}\right) \rightarrow T(R, n)$ given by

$$
f\left(a_{0}+a_{1} x+\ldots+a_{n-1} x^{n-1}+\left(x^{n}\right)\right)=\left(a_{0}, a_{1}, \ldots, a_{n-1}\right),
$$

where $\left(x^{n}\right)$ is ideal in $R[x]$ generated with $x^{n}$, and $\left(a_{0}, a_{1}, \ldots, a_{n-1}\right)$ is a breaf representation for a matrix from $T(R, n)$. Therefore $T(R, n)$ is central Armendariz ring. 
We end this section with our result from [3], in which we give sufficient condition for the power series ring $R[[x ; \sigma]]$ to be reduced.

Theorem 3.2 If an endomorphism $\sigma$ of a reduced ring $R$ satisfies so called compatibi-lity condition: $a \sigma(b)=0 \Leftrightarrow a b=0$, then the power series ring $R[[x ; \sigma]]$ is reduced.

Proof. Let $f(x)=\sum_{i=0}^{\infty} a_{i} x^{i}$ and $(f(x))^{2}=0$. It is clear that $a_{0}^{2}=0$, so that $a_{0}=0$. Now from the compatibility condition $a_{1} \sigma\left(a_{1}\right)=0$ implies $a_{1}^{2}=0$, but since $R$ is reduced we have $a_{1}=0$. By induction argument we have $a_{i}=0$ for all $i$. This means that $f(x)=0$ and so $R[[x ; \sigma]]$ is reduced.

Without compatibility condition the previous theorem is not true. Since for the ring $R=Z_{2} \oplus Z_{2}$ and $\sigma$ defined by $\sigma(a, b)=(b, a)$, it is easy to check that $R[[x ; \sigma]]$ is not reduced. Observe that $(1,0)(0,1)=(0,0)$ but $(1,0) \sigma(0,1) \neq(0,0)$.

\section{REFERENCES}

[1] N. Agayev, G. Gungoroglu, A. Harmanci and S. Halicioglu, "Central Armendariz Rings", Bull. Malays. Math. Sci. Soc., 2(34(1)), 137-145 (2011).

[2] M. Rege, and S. Chhawchharia, "Armendariz rings", Proc. Japan Acad. Ser. A. Math. Sci., 73, 14-17 (1997).

[3] D. Jokanović, "Properties of Armendariz rings and weak Armendariz rings", Pubications de l'Institute Mathematique, Nouvelle serie, 85(99), 131-137 (2009).

[4] L. Ouyang, "Extensions of generalized $\alpha$-rigid rings", International Journal of Algebra, 3, 105-116 (2008).

[5] W. Chen and W. Tong, "On skew Armendariz and rigid rings", Houston Journal of Mathematics, 22, (2007).

Received November 15, 2019 